\title{
Contemporary protease inhibitors and cardiovascular risk
}

Jens Lundgren ${ }^{1}$, Amanda Mocroft ${ }^{2}$, Lene Ryom ${ }^{1}$,

1, CHIP/PERSIMUNE, department of infectious diseases, Rigshospitalet, University of Copenhagen, Denmark

2, Centre for Clinical Research, Epidemiology, Modelling and Evaluation (CREME), Institute for Global Health, University College London, London, UK

\section{Corresponding Author}

Professor Amanda Mocroft

Centre for Clinical Research, Epidemiology, Modelling and Evaluation (CREME)

Institute for Global Health

University College London

Rowalnd Hill St

London

NW3 2PF 
Abstract (200 words max)

Purpose of review: Review the evidence linking use of HIV protease inhibitors with excess risk of cardiovascular disease (CVD) in HIV+ populations.

Recent findings: For the two contemporary most frequently used protease inhibitors, darunavir and atazanavir (both pharmacologically boosted with ritonavir [/r]), darunavir/ $r$ has been shown to be associated with increased CVD risk. The effect is cumulative with longer exposure increasing risk and an effect size comparable to what has been observed for previously developed protease inhibitors. Biological mechanisms may be overlapping and include perturbed lipid metabolism and accumulation of cholesterol derivatives within macrophages. Conversely, atazanavir/ $r$ has not been shown to be associated with CVD, possible due its ability to increase cardioprotective bilirubin levels.

Summary: Evidence linking protease inhibitors to CVD is based on observational studies only, whereas possible plausible biological explanation are well established and derived from randomised trials and controlled experiments. Given the possible association with clinical disease, a conservative approach to apply the data in daily practise is proposed which is focused on individualisation of care based on underlying risk of CVD.

\section{Introduction}

Recent guidelines indicate that antiretroviral therapy (ART) should be offered to all HIV+ persons irrespective of the stage of the infection. The benefit to the individual in early stages of disease comprise a reduced risk of opportunistic infections, invasive bacterial infections, tuberculosis, and cancer $(1,2)$. Additionally, the risk of onward HIV transmission via sex, placental or infected needles is virtually nil in persons with suppressed HIV viraemia $(3,4)$. Due to marked viral diversity within the body soon after initial infection, the probability of genetic variants rendering the virus less susceptible for a given antiretroviral drug is high. This can be overcome with combining agents, ensuring a genetic barrier of at least three and preferable four active antiretrovirals (5).

Protease inhibitors (PIs) provide a high genetic barrier and were among the first developed in the second wave of antiretroviral drug discovery in 1994-1997. Of the first generation Pls, indinavir and saquinavir were the two most frequently used. Pill burden in HIV+ persons was reduced and pharmacokinetics significantly improved by blocking the P450 enzyme system with ritonavir $(/ r)(6,7)$. Lopinavir/r was introduced six years later and was preferred because of better tolerability $(7,8)$. Atazanavir/ $\mathrm{r}$ and darunavir/ $r$ were subsequently introduced and remain the favoured PIs as part of a contemporary ART regimen $(9,10)$. Atazanavir/ $r$ may lead to unharmful but cosmetically unacceptable icterus in a few individuals. Darunavir/ $r$ causes gastrointestinal symptoms, but more rarely than seen in those treated with lopinavir/r (11,12). 


\section{ART and CVD}

It remains uncertain whether ART reduces the risk of CVD. Data from the randomised controlled trial SMART (13), assessing continued versus intermittent use of ART, suggested that the risk may be lower for those using ART continuously, although this effect was of borderline significance $(p=0.05)(14)$. Conversely, cohort studies performed among persons using the first generation ARVs as part of ART suggested a cumulative effect, with risk increasing linearly as duration of ART increased (figure $1(15,16)$ ). Of note, in the first paper describing this finding in observational data, (figure 1), the risk of CVD in those not yet having started ART was the lowest, indicating that most of the CVD events seen in HIV+ populations were associated with use of ART and not untreated HIV. Subsequent research until 2007-2010 indicated that this adverse effect was mainly associated with use of the PIs available at that time (16-19). Each of the PIs used were associated with a gradually increasing CVD risk, so that the risk was not simply confined to those ever exposed, but increased as exposure increased, leading to around a 50\% increase in CVD incidence after five years of exposure.

The range of the relative increased risk over five year of exposure in these studies was between $50 \%$ and $100 \%$. Of note, the observational cohorts from where the data were derived from have been progressively more extensively treated with anti-dyslipidemic drugs over calendar time. This may at least in part be reflective of a reaction to the early reporting of excessive CVD risk by the responsible clinicians. Regardless, and if assuming that part of the mechanism from using PI's is how they adversely affect lipid metabolism (see section below), this more aggressive approach to managing dyslipidaemia may likely have counteracted some of the risk associated with using PI's. In support of this, the association was more pronounced in the earliest of the studies (15) than in subsequent reporting (16.19).

Population based studies comparing risk of CVD in HIV+ versus HIV- populations also suggest a persistent elevated risk of CVD associated with HIV status (20-24). It is intriguing that adjustment for traditional CVD risk factors such as hypertension, smoking, dyslipidaemia and diabetes does not remove the CVD association with HIV status, suggesting that a higher prevalence of such traditional risk factors in the HIV+ population does not explain this finding. Recently, Klein and colleagues (24) published data suggesting some attenuation of the excess CVD risk over calendar time. This attenuation was potentially related to switches in approach to treat HIV using more CVD friendly drugs and being progressively more aggressive in treating traditional CVD risk factors over time.

It is possible that the excess risk of CVD from using ART may have underestimated the beneficial effects of ART on the risk of CVD in the SMART study (14) referred to above. SMART was conducted during the era when antiretrovirals associated with an increased CVD risk were more widely used, and had that not been the case, it is plausible that that the group randomised to continued use of ART may have had even lower rates of CVD. Importantly, given the design of the SMART trial and the focus on persons that had been on ART for several years, there was a sizable group of relatively older persons in the trial which were at higher risk of CVD. In the randomised START trial, performed between 2010 and 2015 (2), there were no signs that timing of initiation of ART (immediately versus deferred) affected arterial elasticity (a proxy for atherosclerosis)(25). The number of CVD events was however too few to assess the impact of ART or type of ART directly. The trial focused on contemporary persons initiating ART, individuals were relatively young in CVD terms, and the primary objective was to assess whether all persons should be offered access to ART irrespective of the stage of their infection. There was strong evidence that offering ART to all was of clinical benefit. Given these results, the question of whether ART benefits persons in terms of their CVD risk or not has become less important. Conversely, the central question in relation to HIV care moving forward, and since the HIV population is aging and HIV is becoming a chronic disease associated with morbidity, is whether contemporarily used PIs accelerate CVD or not. 
Analysis of pooled data from 19 Janssen-sponsored clinical trials did not indicate an increased risk of CVD events with darunavir/r use over treatment durations of up to six years, but the study was limited to 5721 persons followed for a median duration of approximately two years (26). In contrast, Ryom et al. (27) recently presented data from one large observational study which for the first time indicated that darunavir/ $r$ was associated with a gradually increasing risk of CVD over five years of exposure similar to the earlier developed PIs. In contrast, atazanavir/ $r$ was not associated with CVD. This finding suggests, for the first time, that PIs as a class is not associated with excess risk of CVD. The key questions derived from this study is whether darunavir/ $r$ indeed has a CVD risk profile comparable to that seen for first and second generation PIs and furthermore, why atazanavir/ $\mathrm{r}$ is not associated with what otherwise appears to be class effect on CVD risk?

To address this, it is important to critically evaluate the evidence that support a causal link between use of PIs and increased risk of CVD.

\section{Quality of evidence linking PIs to CVD}

No adequately powered randomised trial has yet been conducted to assess the causative relationship between contemporary PIs and CVD. Pivotal trials in HIV drug discovery are all powered to assess viral efficacy, and the sample size to establish this is sizably lower than required for studying clinical endpoints. The SMART (13) and START (2) trials, addressing strategic questions for whether to use ART continuously or not or to initiate ART in early HIV stages or not, were also not designed to directly address this.

As such, it is data derived from careful analysis of adequately powered cohort studies, with their intrinsic limitations to establish causation, that are required to provide evidence to support the causal link. Clearly there are many limitations and bias associated with cohort studies, including most notably when assessing associations with antiretrovirals, the potential for confounding by indication. This arises when the decision to treat a specific individual with a given CART regimen is associated with the outcome. For example, a person at perceived high risk of CVD, according to their physician, would be treated with a different ART regimen to one not thought to be at high risk of CVD. Even with a wide range of information on potential confounding variables to adjust for, this bias may still play a role. None the less, attributes from these cohort study results with the specificity to darunavir/r, but not atazanavir/ $r$ and the biological gradient render it plausible that the detected association maybe causal. No study to date has been adequately powered to investigate if the observed association between darunavir/ $r$ and CVD declines when individuals are switched off darunavir/r, but if this proves to be the case it will be an important additional evidence to support causality of the association.

The association between PIs and CVD is reproducible and seen consistently in a number of cohorts and when assessing different scenarios (15-19). This to some extent reduces - albeit does not remove - the risk that unknown or unmeasured confounders that could not be adjusted for might explain the association. Further, the association is characterised as a gradual increase, suggesting a dose-response effect and a slowly increasing risk as cumulative exposure increases (figure 1); a similar shape of the association was also seen for the darunavir/r signal (27). If causal, this implies that PIs gradually worsen the underlying arterial pathology. There are at least two plausible mechanisms by which this may occur.

PIs perturbs lipid metabolism and leads to a pro-atherogenic state. This effect is demonstrated in multiple randomised controlled trials (6-12). Table 1 shows data from a recent publication from the START trial by Baker et al (28); of note, total and LDL cholesterol levels during follow-up were increased for those starting Pl's immediately versus deferred. Molina et al. also demonstrated this effect in relation to darunavir/ $r$ 
when compared to a drug (dolutegravir) from the integrase inhibitor drug class which are known not to affect lipid metabolism. (11). Of note, adjustment for cholesterol levels after starting PIs in observational studies attenuates (but does not remove) the association between PI exposure and CVD risk $(17,19)$. This attenuation of the association however was not seen for the darunavir/ $r$ signal (27). This body of evidence would suggest that although PIs perturb lipid metabolism, and that this perturbation may partly be responsible for the association between excess risk of CVD for some PI's, the potential for lipid perturbation may not be the same for all PIs and therefore is not a consistent plausible biological explanation The other plausible explanation was observed in 2003 (29). Pls upregulate the accumulation of cholesteryl esters in macrophages via induction of CD36; an effect that oestrogen attenuates (30). Macrophages of course are central in the formation of atherosclerotic plaques. PIs might further inhibit apoptosis, which could potentially alter the turnover of plaque macrophages. While the role of plaque apoptosis is debated and enhanced apoptosis in certain cells might alter plaque stability, inhibition may also lead to retention of harmful macrophage phenotypes (31).

Atazanavir/r share the two mechanisms outlined above with darunavir/r, yet the $D: A: D$ study could not find any evidence of an increased risk of CVD associated with atazanavir/r (27). It was been proposed that the elevation of bilirubin caused by atazanavir/ $r$ may reduce any association between atazanavir/ $r$ and increased CVD risk (32-34). Marconi and colleagues reported that higher bilirubin levels were associated with lower risk of CVD independent of other confounders (35).

It is questionable whether any sufficiently powered randomised clinical trial will ever be conducted to confirm the causal relationship between darunavir/ $\mathrm{r}$ and other PIs and excess risk of CVD. This continued uncertainty needs to be incorporated into clinical management of HIV+ persons and considered alongside the other risks and benefits associated with ART. It would seem reasonable to approach the association between darunavir/ $\mathrm{r}$ and CVD risk conservatively, taking into account both relative versus absolute risk as well as benefits and risks of ART.

\section{Relative versus absolute risk}

The key study findings reporting an association between PIs and CVD expresses their result in relative terms. That is, the risk of CVD was increased by e.g. 50\%-100\% after five years of use. However, if the person's underlying risk of CVD is very low, a 50\%-100\% increase in risk is minimal and the person will remain at a low risk of CVD whether or not they are exposed to PIs. The opposite is true if the underlying risk is high. There are now reliable and validated risk algorithms able to estimate the underlying risk (34). These risk algorithms are critical to use as part of daily practise to be able to individualise care. Not only are they able to differentiate risk of contracting adverse drug reactions, such as CVD, but they also focus attention to the factors in the algorithms that influence the underlying risk including those being potentially modifiable including other types of antiretroviral drugs (36).

\section{Balancing benefits and harm}

The overall philosophy of practising medicine is to provide care for the individual to optimise the chance of benefit by weighing and potentially reducing risk for causing harm. Quantification of risk and harm, as outlined in the section above, is hence critically important to perform. Also, the benefits from using ART (as opposed to not doing so) weigh very heavily in favour of using ART; the introduction of combination ART has led to a $90 \%$ reduction in morbidity and mortality associated with HIV (37). For PIs, given the high genetic barrier for drugs in this class (in particular darunavir/r), these drugs are an important component of the accessible antiretroviral drugs in situations where patients have already acquired drug resistance or in situations where the risk of variable adherence (and hence acquisition of drug resistance) is high. 


\section{Conclusions}

PIs remains an integral part of ART, in particular due to their ability to maintain full control of viral replication in persons harbouring HIV resistant to several other of the available antiretroviral drugs. A small increased risk of CVD, which increases with increased length of exposure, may be an adverse effect from using most drugs from within this drug class, other than atazanavir/r. The evidence basis for this association is based largely on analyses from observational studies with their inherited limitations and inability to establish causality. It is plausible that the effect is driven by perturbation of cholesterol metabolism. Additional research, including adequately powered randomised trials, is required to further substantiate the evidence base for using this important drug class used as part of ART.

Key words: Cardiovascular disease, protease inhibitors, HIV

Declaration of conflict of interest

JDL's and LR' s institution has received research grant support from Gilead Sciences Inc., ViiV Healthcare, Merck \& Co Inc. and Janssen Pharmaceuticals. AM has received honoraria, lecture fees, and/or travel support from Gilead Sciences Inc., ViiV Healthcare, Merck \& Co Inc., Pfizer, Boehringer Ingelheim and Bristol Myers Squibb. This study was supported by the Danish National Research Foundation [grant no 126]. 
References:

1. Danel C, Moh R, Gabillard D, et al. A Trial of Early Antiretrovirals and Isoniazid Preventive Therapy in Africa. N Engl J Med. 2015 Aug 27;373(9):808-22.

2. Lundgren JD, Babiker AG, Gordin F, Emery S, Grund B, Sharma S, et al. Initiation of antiretroviral therapy in early asymptomatic HIV infection. N Engl J Med 2015; 373:795-807.

3. Cohen MS, Chen YQ, McCauley M, et al. Antiretroviral Therapy for the Prevention of HIV-1 Transmission. N Engl J Med. 2016 Sep 1;375(9):830-9.

4. Rodger AJ, Cambiano V, Bruun T, et al. Sexual activity without condoms and risk of HIV transmission in serodifferent couples when the HIV-positive partner is using supportive antiretroviral therapy. JAMA 2016; 316:171-181.

5. **EACS Guidelines version 9.0. http://www.eacsociety.org/files/guidelines_9.0-english.pdf (accessed 4 November 2017). Updated guidelines on clinical management of HIVpositive persons including non-infectious co-morbidities. Can be downloaded as an app for everyday use.

6. Dragsted UB, Gerstoft J, Pedersen C, et al. Randomized trial to evaluate indinavir/ritonavir versus saquinavir/ritonavir in human immunodeficiency virus type 1-infected patients: the MaxCmin1 Trial. J Infect Dis. 2003 Sep 1;188(5):635-42.

7. Walmsley $\mathrm{S}$, Bernstein $B$, King $M$, et al. Lopinavir-ritonavir versus nelfinavir for the initial treatment of HIV infection. N Engl J Med. 2002 Jun 27;346(26):2039-46.

8. Dragsted UB, Gerstoft J, Youle M, et al. A randomized trial to evaluate lopinavir/ritonavir versus saquinavir/ritonavir in HIV-1-infected patients: the MaxCmin2 trial. Antivir Ther. 2005;10(6):735-43

9. DeJesus E, Rockstroh JK, Henry K, et al. Co-formulated elvitegravir, cobicistat, emtricitabine, and tenofovir disoproxil fumarate versus ritonavir-boosted atazanavir plus co-formulated emtricitabine and tenofovir disoproxil fumarate for initial treatment of HIV-1 infection: a randomised, doubleblind, phase 3, non-inferiority trial. Lancet. 2012 Jun 30;379(9835):2429-38.

10. Madruga JV, Berger D, McMurchie M, et al. Efficacy and safety of darunavir-ritonavir compared with that of lopinavir-ritonavir at 48 weeks in treatment-experienced, HIV-infected patients in TITAN: a randomised controlled phase III trial. Lancet. 2007 Jul 7;370(9581):49-58.

11. *Molina JM, Clotet B, van Lunzen J et al. Once-daily dolutegravir versus darunavir plus ritonavir for treatment-naive adults with HIV-1 infection (FLAMINGO): 96 week results from a randomised, open-label, phase 3b study. Lancet HIV. 2015 Apr;2(4):e127-36. doi: 10.1016/S2352-

3018(15)00027-2. An example of a randomised study of several laboratory outcomes including lipids comparing a contemporary PI (darunavir/ritonavir) with a drug from the integrase inhibitor class (dolutegravir) which do not affect lipid metabolism.

12. Orkin C, DeJesus $E$, Khanlou $H$, et al. Final 192-week efficacy and safety of once-daily darunavir/ritonavir compared with lopinavir/ritonavir in HIV-1-infected treatment-naive patients in the ARTEMIS trial. HIV Medicine 2013 Jan;14(1):49-59. 
13. El-Sadr WM, Lundgren J, Neaton JD, et al. CD4+ count - guided interruption of antiretroviral treatment. N Engl J Med 2006; 355:2283-2296.

14. Phillips AN, Carr A, Neuhaus J, et al. Interruption of antiretroviral therapy and risk of cardiovascular disease in persons with HIV-1 infection: exploratory analyses from the SMART trial. Antivir Ther. 2008;13(2):177-87.

15. Friis-Moller N, Sabin CA, Weber R, et al. Combination antiretroviral therapy and the risk of myocardial infarction. The New England journal of medicine 2003 Nov 20;349(21):1993-2003.

16. Mary-Krause M1, Cotte L, Simon A, Partisani M, Costagliola D. Increased risk of myocardial infarction with duration of protease inhibitor therapy in HIV-infected men. AIDS. $2003 \mathrm{Nov}$ 21;17(17):2479-86.

17. Friis-Møller N, Reiss $P$, Sabin CA, et al. Class of antiretroviral drugs and the risk of myocardial infarction. N Engl J Med. 2007 Apr 26;356(17):1723-35

18. D'Ascenzo F, Cerrato E, Biondi-Zoccai G, Moretti C, Omedè P, Sciuto F, et al. Acute coronary syndromes in human immunodeficiency virus patients: a meta-analysis investigating adverse event rates and the role of antiretroviral therapy. Eur Heart J 2012;33(7):875-80.

19. Worm SW, Sabin C, Weber R, et al. Risk of myocardial infarction in patients with HIV infection exposed to specific individual antiretroviral drugs from the 3 major drug classes: the data collection on adverse events of anti-HIV drugs (D:A:D) study. The Journal of infectious diseases $2010 \mathrm{Feb}$ 1;201(3):318-30.

20. Triant $\mathrm{V}$ a, Lee $\mathrm{H}$, Hadigan $\mathrm{C}$, Grinspoon SK. Increased acute myocardial infarction rates and cardiovascular risk factors among patients with human immunodeficiency virus disease. J Clin Endocrinol Metab [Internet]. 2007;92(7):2506-12.

21. Obel N, Thomsen HF, Kronborg G, Larsen CS, Hildebrandt PR, Sorensen HT, et al. Ischemic Heart Disease in HIV-Infected and HIV-Uninfected Individuals: A Population-Based Cohort Study. Clin Infect Dis 2007;44(12):1625-31.

22. Lang S, Mary-Krause $M$, Cotte L, et al. Increased risk of myocardial infarction in HIV-infected patients in France, relative to the general population. AIDS. 2010;24:1221-30.

23. Freiberg MS, Chang CC, Kuller LH, et al, HIV infection and the risk of acute myocardial infarction. JAMA Intern Med. 2013 Apr 22;173(8):614-22

24. Klein DB, Leyden WA, Xu L, et al, Declining relative risk for myocardial infarction among HIV-positive compared with HIV-negative individuals with access to care. Clin Infect Dis. 2015 Apr $15 ; 60(8): 1278-80$.

25. ${ }^{* *}$ Baker JV, Hullsiek KH, Engen NW, et al. Early Antiretroviral Therapy at High CD4 Counts Does Not Improve Arterial Elasticity: A Substudy of the Strategic Timing of AntiRetroviral Treatment (START) Trial. Open Forum Infect Dis. 2016 Oct 8;3(4):ofw213. Primary report of a substudy in START, assessing whether arterial elasticity was affected by early versus deferred initiation of ART. Arterial elasticity was used as a proxy for arterial wall disease based on studies in the general population. The strategy of using ART did not affect this outcome. 
26. * Opsomer M, Dimitrova D, Verspeelt J, et al. Evaluation of Cardiovascular Disease (CVD) Risk in HIV1-infected Patients Treated With Darunavir/Ritonavir (DRV/r). 11th Annual American Conference for the Treatment of HIV, 2017, Dalles (poster 18). A presentation of data from randomised controlled trials conducted as part of the clinical development program for darunavir by the marketing authority holder. Lauched in response to an abstract from the D:A:D study (ref 28). Of note, these trials were designed to assess laboratory outcomes with HIV viral load being the primary, and hence were not powered to assess the clinical risk of cardiovascular disease.

27. ${ }^{* *}$ Ryom L, Lundgren JD, El-Sadr W, et al. Association between Cardiovascular Disease \& Use of Contemporary Protease Inhibitors: The D:A:D Study. Conference on Retroviruses and Opportunistic Infections (CROI) 2017, Seatle, abstract 128LB. A late-breaker presentation associating duration of exposure of two protease inhibitors (darunavir and atazanavir - both ritonavir boosted) and cardiovascular disease risk; a significant association was found the former but not with the latter protease inhibitor.

28. **Baker JV, Sharma S, Achhra AC, et al. Changes in Cardiovascular Disease Risk Factors With Immediate Versus Deferred Antiretroviral Therapy Initiation Among HIV-Positive Participants in the START (Strategic Timing of Antiretroviral Treatment) Trial. J Am Heart Assoc. 2017 May 22;6(5). pii: e004987. An analysis of the START trial (comparing immediate versus deferred ART) assessing how these two strategies of using ART affects traditional cardiovascular risk factors. Importantly, immediate ART did not dampen the risk of cardiovascular risk factor profile as otherwise stipulated in several guidelines and commentaries in the years preceding the release of the findings from START.

29. Dressman J, Kincer J, Matveev SV, et al. HIV protease inhibitors promote atherosclerotic lesion formation independent of dyslipidemia by increasing CD36-dependent cholesteryl ester accumulation in macrophages. The Journal of clinical investigation 2003 Feb;111(3):389-97.

30. Wilson ME, Sengoku T, Allred KF. Estrogen prevents cholesteryl ester accumulation in macrophages induced by the HIV protease inhibitor ritonavir. J Cell Biochem. 2008 Apr 1;103(5):1598-606.

31. Badley AD, Pilon AA, Landay A, Lynch DH. Mechanisms of HIV-associated lymphocyte apoptosis. Blood 2000; 96(9);2951-2964.

32. Vogel ME, Idelman G, Konaniah ES, Zucker SD. Bilirubin Prevents Atherosclerotic Lesion Formation in Low-Density Lipoprotein Receptor-Deficient Mice by Inhibiting Endothelial VCAM-1 and ICAM-1 Signaling. J Am Heart Assoc 2017 Apr 01;6(4).

33. Kunutsor SK, Bakker SJ, Gansevoort RT, Chowdhury R, Dullaart RP. Circulating total bilirubin and risk of incident cardiovascular disease in the general population. Arteriosclerosis, thrombosis, and vascular biology 2015 Mar;35(3):716-24.

34. Marconi VC, So-Armah K, Tate J, et al. Bilirubinemia prevents cardiovascular disease for HIV+ and HIV- individuals. Conference on Retroviruses and Opportunistic Infections (CROI) 2017, Seatle, abstract 127.

35. ${ }^{* *}$ Friis-Moller N, Ryom L, Smith C, et al. An updated prediction model of the global risk of cardiovascular disease in HIV-positive persons: The Data-collection on Adverse Effects of Anti-HIV Drugs (D:A:D) study. Eur J Prev Cardiol 2016 Jan;23(2):214-23. Updated version of the D:A:D 
cardiovascular risk predictor - the algorithm can be accessed for individual patient assessment via the following link: https://www.chip.dk/Tools-Standards/Clinical-risk-scores

36. Sabin CA, Reiss P, Ryom L, et al. Is there continued evidence for an association between abacavir usage and myocardial infarction risk in individuals with HIV? A cohort collaboration. BMC Med 2016;14:61.

37. Mocroft A, Vella S, Benfield TL, et al. Changing patterns of mortality across Europe in patients infected with HIV-1. EuroSIDA Study Group. Lancet. 1998 Nov 28;352(9142):1725-30. 
Table 1. Lipid differences in the START study comparing persons starting immediate versus deferred on drugs from one drug (efavirnez) and drugs from protease inhibitors and integrase inhibitor drug classes (28).

\begin{tabular}{|c|c|c|c|}
\hline & \multicolumn{3}{|l|}{ Pre-specified ART } \\
\hline & $\operatorname{EFV}(n=3,516)$ & $\mathrm{PI}(n=815)$ & INSTI (n=183) \\
\hline Measure & $\begin{array}{l}\text { I-D a } \\
\text { Mean Diff. }[95 \% \mathrm{Cl}]\end{array}$ & $\begin{array}{l}\text { I-D a } \\
\text { Mean Diff. }[95 \% \mathrm{Cl}]\end{array}$ & $\begin{array}{l}\text { I-D }{ }^{a} \\
\text { Mean Diff. }[95 \% C l]\end{array}$ \\
\hline Total-C (mg/dL) & $13.2[11.5,14.9]^{*}$ & $8.7[5.2,12.1]^{*}$ & $-2.4[-10.1,5.3]$ \\
\hline LDL-C (mg/dL) & $6.5[5.0,7.9]^{*}$ & $3.7[0.7,6.7]$ & $-1.1[-7.8,5.7]$ \\
\hline HDL-C (mg/dL) & $5.8[5.2,6.4]^{*}$ & $2.2[0.9,3.5]^{*}$ & $0.4[-2.5,3.4]$ \\
\hline Total-C:HDL-C & $-0.2[-0.2,-0.1]^{* *}$ & $-0.0[-0.2,0.1]$ & $-0.2[-0.5,0.2]$ \\
\hline BMI $\left(\mathrm{kg} / \mathrm{m}^{2}\right)$ & $-0.3[-0.4,-0.2]^{* *}$ & $-0.1[-0.2,0.1]$ & $-0.0[-0.4,0.4]$ \\
\hline
\end{tabular}


Figure 1. Myocardial infarction by exposure to antiretroviral therapy* used between 2000-2002 (15).

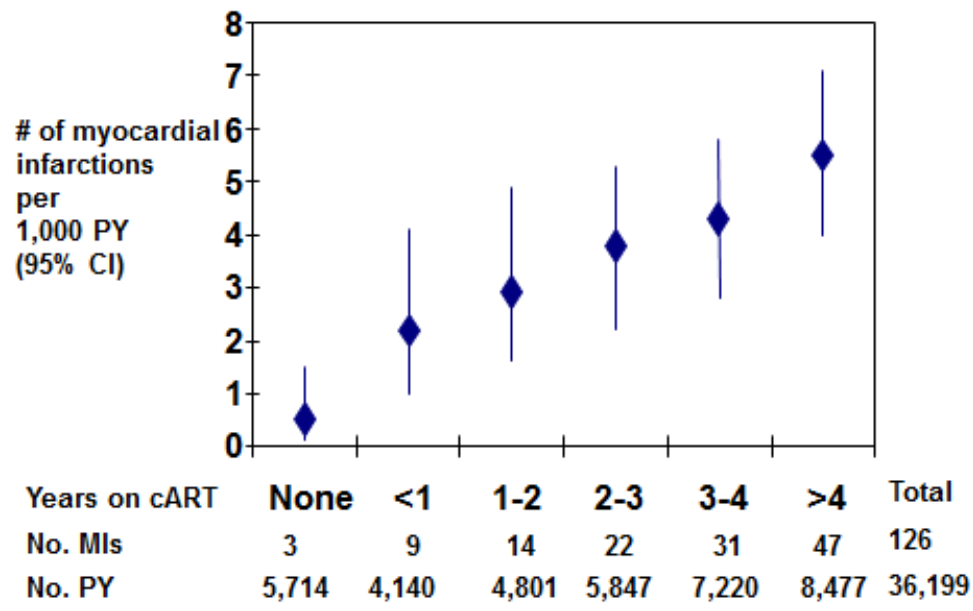

*: Most antiretroviral therapy used at the time included one of the first generation protease inhibitors. Note, the gradually increasing with longer use ( $27 \%$ increased per year of use). 